\title{
Impacto del asma en escolares de dos centros de salud primaria
}

\author{
CLAUDIA VELÁSTEGUI ${ }^{1}$, PAULA PÉREZ-CANTO ${ }^{1}$, \\ VÍCTOR ZÁRATE², DEIDYLAND ARENAS ${ }^{3 a}$, PAMELA SALINAS ${ }^{4 b}$, \\ GLADYS MORENO ${ }^{1}$, FRANCISCO PRADO ${ }^{4,5}$
}

\section{Impact of asthma among primary attention children}

Background: Asthma is one of the chronic diseases that generates an impact on quality of life and major health care expenses. Aim: To evaluate the impact of asthma on primary atenttion expenses, the health related quality of life (HRQOL) and the sanitary resources use. Material and Methods: Follow up of a cohort of 56 asthmatic children aged 6 to 14 years (56\% women), during 12 months. The severity and the impact of the disease on HRQOL was assessed using the International Study of Asthma and Allergies in Childhood (ISAAC) video questionnaire and the Pediatric Asthma Quality of Life Questionnaire (PAQLQ) respectively. Costs were derived from the number of medical attentions, hospital admissions, medication requirements, school absenteeism among patients and work absenteeism among caregivers. Results: Patients required twice the number of medical attentions than the general population. Fourteen percent of children had severe asthma. Compared with subjects without severe asthma, the latter required more attentions (3.8 and 9.7 attentions per year respectively, $p<0.01)$, had a lower PAQLQ score $(4.1 \pm 0.11$ and $5.2 \pm 1$ respectively, $p<0.05$ ) and a higher school absenteeism (10 and 27 days per year respectively, $p<0.01)$. Medication use was not different between patients with and without severe asthma. The yearly cost per patient was \$106,550 (Chilean pesos). Conclusions: Asthma has a great impact on HRQOL and the use of primary attention resources, specially those children with severe asthma.

(Rev Med Chile 2010; 138: 205-212).

Key words: Asthma; Child; Quality of life.

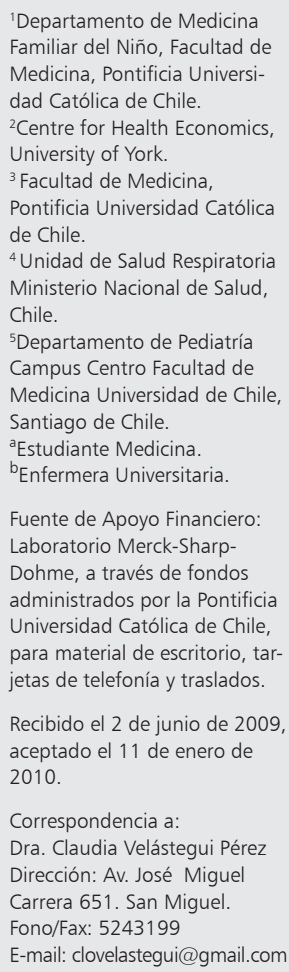

$\mathrm{E}$ l asma es una enfermedad pediátrica cuya prevalencia ha aumentado significativamente en todo el mundo ${ }^{1-5}$. Determina impacto económico en los pacientes, sistemas sanitarios y sociedad ${ }^{1,6,7}$. Produce deterioro de la calidad de vida relacionada con salud (CVRS) en niños y adolescentes ${ }^{1-4}$, asociado con limitación de las actividades diarias, físicas y lúdicas. Interfiere con el sueño, rendimiento académico y condiciona ausentismo escolar y laboral de los padres $^{1,8-10}$.

La carga económica y CVRS empeoran por exacerbaciones que requieren consultas no progra- madas, atención de urgencia y hospitalizaciones; pudiendo aminorarse con estrategias programáticas costo-efectivas, como educación y terapia antiinflamatoria ${ }^{2,11-15}$. El asma produce costos en los sistemas de salud y familias. Los costos directos comprenden hospitalizaciones, consultas no programadas en la atención primaria en salud (APS) y servicios de urgencia, uso de medicamentos, exámenes diagnósticos y procedimientos. Los costos indirectos más importantes incluyen días de ausentismo laboral y escolar ${ }^{2,9}$. Existen estudios del impacto del asma en países desarrollados ${ }^{6,16}$, pero existen pocos datos acerca de éste en países 
latinoamericanos, información esencial para desarrollar mejores estrategias locales.

La prevalencia del asma infantil en Chile fluctúa entre $7,3 \%$ y $16,5 \%{ }^{5,17}$. En la mayoría de los casos es leve o moderada. En el sistema público de salud, los niños y adolescentes con asma leve se controlan en la APS; los asmáticos moderados, en APS y atención secundaria; y los asmáticos severos, preferentemente en atención secundaria. En Chile no existen estudios que caractericen conjuntamente carga económica y repercusión en CVRS del asma infantil. En consecuencia, sin datos nacionales, la evaluación del impacto de las estrategias actuales o futuras para el control del asma en este grupo, está claramente limitada. El objetivo de este estudio es conocer el impacto del asma en la utilización de recursos sanitarios y en CVRS de niños y adolescentes asmáticos entre 6 y 14 años, atendidos en dos centros de APS pertenecientes a la zona sur-oriente de la Región Metropolitana (RM).

\section{Pacientes y Método}

El estudio fue diseñado como un análisis descriptivo transversal y de seguimiento durante 12 meses de una cohorte de escolares asmáticos. Se realizó en dos Centros de Salud Familiar (CESFAM) de la comuna de Puente Alto, Santiago: Madre Teresa de Calcuta y Bernardo Leighton.

Se identificaron pacientes entre 6 y 14 años inclusive, fueron reclutados a través de una invitación telefónica o citación domiciliaria, e ingresados al estudio después de obtener consentimiento informado de sus cuidadores.

Para categorizar la severidad del asma se uso un videocuestionario propuesto por el International Study of Asthma and Allergies in Childhood (ISAAC) para estudiar la prevalencia de asma en niños ${ }^{18,19}$. En la primera reunión se aplicó el videocuestionario que tiene una duración de diez minutos, incluye 5 secuencias de síntomas asmáticos en personas jóvenes que son: sibilancias en reposo, sibilancias tras el ejercicio, despertar nocturno con sibilancias, despertar nocturno con tos, y crisis grave de asma con dificultad para respirar en reposo o "episodio severo".

Después de cada secuencia se pidió a los niños que respondieran por escrito a las preguntas sobre el video. Se solicitó que especificaran si su respiración había sido alguna vez como la persona del vídeo, y si así fue, si les había ocurrido en el último año con frecuencia semanal. La severidad del asma fue categorizada según este instrumento en asma severo y no severo, siendo severos aquellos pacientes que habían presentado un "episodio severo" de asma agudo en el último año con frecuencia semanal.

Como instrumento para el seguimiento se utilizó un cuaderno de registro elaborado en conjunto con el Departamento de Salud Pública y Pediatría de la Pontificia Universidad Católica de Chile, piloteados en ambos CESFAM. Éste consistió en 12 cartillas para registrar mensualmente: presencia de síntomas (tos nocturna, silbido al pecho, tos con ejercicio), número de consultas no programadas en APS y servicios de urgencia, días de hospitalización, uso de medicamentos antiasmáticos y antialérgicos, radiografías, número de sesiones de kinesioterapia, días de ausentismo escolar y de ausentismo laboral del cuidador. Éste último debía, además, guardar los comprobantes de atención entregados en los servicios de urgencia y epicrisis.

Las cartillas mensuales fueron recolectadas trimestralmente. Se confirmó la concordancia de la información reportada por el cuidador, cotejándola con la información registrada en las fichas de ambos CESFAM (10\% al azar del total de pacientes).

La CVRS fue evaluada con un cuestionario específico para niños asmáticos (PAQLQ) validado internacionalmente ${ }^{20-22}$, en su versión en español ${ }^{23}$ y usado previamente en Chile ${ }^{24}$. Éste consta de 23 preguntas, cada una se puntúa según una escala de Likert de 1 a 7. El puntaje 1 representa la máxima afectación y 7 la mínima. Las preguntas se agrupan para obtener 3 dimensiones: síntomas, función emocional y limitación de actividades diarias. Los puntajes obtenidos se expresan en promedios globales y por cada dimensión señalada.

Los costos de medicamentos antiasmáticos, inhaladores de dosis medida (IDM) de salbutamol, corticoides inhalados (CI) y otros fueron obtenidos de una cadena de farmacias nacional. Se utilizaron los valores más bajos disponibles para cada uno. Los costos relacionados a atenciones medicas fueron extraídos del arancel FONASA libre elección $2008^{25}$ y del estudio de la Dirección de Presupuesto 2005 "Programa de Reforzamiento de la Atención Primaria de Salud"26. Para los costos 
Impacto del asma en escolares de dos centros de salud primaria - C. Velástegui et al

indirectos se utilizó como referencia el sueldo base mensual (Ley 20.204), de \$ 144.000 establecido para el año $2008^{27}$ y el costo de la subvención mensual por niño según el Ministerio de Educación (MINEDUC), fijado en $\$ 44.572^{28}$ (Tabla 1 ).

Para el análisis estadístico se utilizó el Programa SPSS versión 13.0. La variabilidad de los estimadores se reporta con desviación estándar (DS). Para las comparaciones entre subgrupos se utilizó t-Student o chi-cuadrado, dependiendo si las variables eran continuas o categóricas. Se consideró estadísticamente significativo un valor $\mathrm{p}<0,05$.

\section{Resultados}

Cuatrocientos noventa pacientes cumplieron los criterios de inclusión. Del total de pacientes,
134 aceptaron participar y $64(47,8 \%)$ completaron el seguimiento (28 niños y 36 niñas), la edad promedio del grupo que finalizó el estudio fue de $9,06 \pm 2,71$ años. No se observaron diferencias significativas en edad $(\mathrm{p}=0,640)$ y género $(\mathrm{p}=$ $0,167)$ y si las hubo en severidad entre los pacientes ingresados al estudio y los que completaron el seguimiento $(\mathrm{p}=0,005)$. Nueve pacientes $(14 \%)$ fueron asmáticos severos y 55 (86\%) asmáticos no severos.

Las características sociodemográficas de los padres y cuidadores se observan en la Tabla 2. El cuaderno de registro fue responsabilidad de la madre en $84,5 \%$ de los pacientes. El promedio anual de consultas fue de 4,8/niño/año. La distribución de ellas según lugar de ocurrencia se muestra en la Tabla 3. Los pacientes con asma severa tuvieron

Tabla 1. Referencia para Análisis de Costos Directos e Indirectos (valores $1^{\circ}$ semestre 2007)

\begin{tabular}{|c|c|c|c|}
\hline Ítem & Fuente & \multicolumn{2}{|c|}{ Costo Total } \\
\hline \multicolumn{4}{|l|}{ Recursos Sanitarios } \\
\hline Consultorio (Atención médica) & DIPRES & $\$$ & 3.040 \\
\hline SAPU (Servicio Atención Primaria de Urgencia) & DIPRES & $\$$ & 1.000 \\
\hline Urgencia & DIPRES & $\$$ & 8.000 \\
\hline Médico Especialista & FONASA I & $\$$ & 9.380 \\
\hline Médico General & FONASA I & $\$$ & 6.120 \\
\hline Radiografías Tórax & FONASA I & $\$$ & 6.130 \\
\hline Hospitalización (día cama) & FONASA I & $\$$ & 4.650 \\
\hline Hospitalización pediátrica (día cama UCI) & FONASA I & $\$$ & 34.730 \\
\hline Hospitalización pediátrica (día cama UTI) & FONASA I & $\$$ & 16.690 \\
\hline Atención Kinesiología Respiratoria & FONASA I & $\$$ & 1.550 \\
\hline \multicolumn{4}{|l|}{ Medicamentos* } \\
\hline Salbutamol & Cadena de Farmacias Nacional & $\$$ & 250 \\
\hline Corticoides inhalatorios & Cadena de Farmacias Nacional & $\$$ & 16.916 \\
\hline Corticoides orales, nasal y endovenoso & Cadena de Farmacias Nacional & $\$$ & 7.033 \\
\hline Antihistamínicos & Cadena de Farmacias Nacional & $\$$ & 2.838 \\
\hline \multicolumn{4}{|l|}{ Ausentismo laboral } \\
\hline Ingreso mínimo mensual & Dirección del Trabajo & $\$$ & 44.000 \\
\hline Ingreso mínimo día (mensual/20) & Dirección del Trabajo & $\$$ & 7.200 \\
\hline \multicolumn{4}{|l|}{ Ausentismo escolar } \\
\hline Subvención escolar mensual & Ministerio de Educación & $\$$ & 44.572 \\
\hline Subvención escolar diaria (mensual/30) & Ministerio de Educación & $\$$ & 1.486 \\
\hline
\end{tabular}

*precio promedio según categoría. 
Impacto del asma en escolares de dos centros de salud primaria - C. Velástegui et al

Tabla 2. Características sociodemográficas de los cuidadores

\begin{tabular}{|llccc|}
\hline Característica sociodemográfica & Variable & (n) & \% & Promedio \\
\hline Relación del entrevistado-paciente & Madre & 49 & 84,5 & 15,5 \\
& Otro & 9 & 23,6 & $10,9 \pm 3,16$ \\
Educación padres/cuidador & $<9$ años & 13 & 54,5 & 21,8 \\
Situación laboral padres/cuidador & $9-12$ años & 30 & 15,5 \\
& $>12$ años & 12 & 79,6 \\
Ingreso personal promedio mensual & Empleado activo & 9 & 5,2 \\
& Dueña de casa & 46 & 50 \\
& Otro & 3 & 23,9 \\
\hline
\end{tabular}

Tabla 3. Frecuencia consultas médicas según lugar de ocurrencia

\begin{tabular}{|lcc|}
\hline Lugar consulta médica & $\begin{array}{c}\text { Promedio } \\
\text { niño/año }\end{array}$ & $\begin{array}{c}\text { \% del } \\
\text { total }\end{array}$ \\
\hline Consultorio & $2,7 \pm 2,9$ & 57 \\
\hline SAPU & $1,3 \pm 5,2$ & 27 \\
\hline Servicio de urgencia & $0,4 \pm 1,1$ & 6,5 \\
\hline Médico general particular & $0,3 \pm 1,0$ & 6,5 \\
Médico especialista particular & $0,1 \pm 0,5$ & 3 \\
\hline Total & $4,8 \pm 3,2$ & 100 \\
\hline
\end{tabular}

mayor número de consultas anuales y no hubo diferencias según género y edad (Tabla 4). El 43\% de los pacientes recibió tratamiento con CI y 77\% salbutamol en IDM. No hubo diferencias según los grupos de severidad (CI: severos $44 \%$ vs $43 \%$ no severo; salbutamol: $78 \%$ severo vs $77 \%$ no-severo).

\section{CVRS, impacto familiar y social}

Los pacientes con asma severa tuvieron menor puntaje global y menor puntaje en la dimensión "síntomas", con diferencias estadísticamente significativas (Tabla 5). La dimensión "limitación de actividades" fue la más afectada en ambos grupos de severidad. Los pacientes con asma severo tuvieron mayor ausentismo escolar $(\mathrm{p}<0,001)$. El
Tabla 4. Consultas médicas. Ocurrencia según género, edad y severidad

\begin{tabular}{|lccc|}
\hline Género & Masculino & Femenino & p \\
& 5,5 & 4,1 & NS \\
Edad & $\mathbf{6 - 9}$ años & $\mathbf{1 0 - 1 4}$ años & \\
& 4,3 & 3,7 & NS \\
Severidad & Severo & No Severo & \\
& 9,7 & 3,8 & $<0,01$ \\
\hline
\end{tabular}

ausentismo laboral de los padres para la totalidad del grupo fue menor a un día y no hubo diferencias según severidad (Tabla 6).

\section{Costos}

La distribución de los costos según severidad se describe en la Tabla 6. El costo total promedio niño/año fue de $\$ 106.550$. El costo directo fue \$ 84.642. El 69\% de los gastos directos corresponde a medicamentos. De ellos 65\% corresponde a CI; IDM de salbutamol $2 \%$ y prednisona $1 \%$; otros medicamentos relacionados al tratamiento de rinitis alérgica (antihistamínicos y corticoides nasales) suman 32\% del gasto total. Los costos directos fueron significativamente mayores en los pacientes 
Tabla 5. Evaluación calidad de vida relacionada con salud (CVRS) según instrumento PAQLQ

\begin{tabular}{|lcccc|}
\hline Evaluación & $\begin{array}{c}\text { Puntaje promedio } \\
\text { población en } \\
\text { seguimiento }\end{array}$ & $\begin{array}{c}\text { Puntaje promedio } \\
\text { población con asma } \\
\text { Dimensión }\end{array}$ & $\begin{array}{c}\text { Puntaje promedio } \\
\text { población con asma } \\
\text { severo }\end{array}$ & $\begin{array}{c}\text { p } \\
\text { pobro }\end{array}$ \\
\hline Puntaje global & $5,05 \pm 1,04$ & $5,23 \pm 0,95$ & $4,09 \pm 0,11$ & $<0,05$ \\
\hline Actividad & $4,39 \pm 0,95$ & $4,43 \pm 0,94$ & $4,24 \pm 1,05$ & $\mathrm{NS}$ \\
\hline Síntomas & $5,49 \pm 1,31$ & $5,65 \pm 1,20$ & $4,21 \pm 1,56$ & $<0,01$ \\
\hline Emociones & $5,83 \pm 1,14$ & $5,92 \pm 1,08$ & $5,42 \pm 1,42$ & $\mathrm{NS}$ \\
\hline
\end{tabular}

Tabla 6. Costos anuales. Distribución según severidad del asma

\begin{tabular}{|c|c|c|c|c|c|c|c|c|}
\hline \multirow[t]{2}{*}{$\begin{array}{l}\text { Tipo de } \\
\text { costo }\end{array}$} & \multirow[t]{2}{*}{ Variable } & \multicolumn{2}{|c|}{$\begin{array}{l}\text { Población en } \\
\text { seguimiento }\end{array}$} & \multicolumn{2}{|c|}{$\begin{array}{c}\text { Población con asma } \\
\text { no severo }\end{array}$} & \multicolumn{2}{|c|}{$\begin{array}{c}\text { Población con asma } \\
\text { severo }\end{array}$} & \multirow[t]{2}{*}{$\mathbf{p}$} \\
\hline & & $\begin{array}{l}\text { Prome- } \\
\text { dio en } \\
\text { pesos } \\
\text { al año }\end{array}$ & $\begin{array}{c}\% \text { del } \\
\text { tipo de } \\
\text { costo }\end{array}$ & $\begin{array}{l}\text { Prome- } \\
\text { dio en } \\
\text { pesos } \\
\text { al año }\end{array}$ & $\begin{array}{l}\% \text { del } \\
\text { tipo de } \\
\text { costo }\end{array}$ & $\begin{array}{c}\text { Prome- } \\
\text { dio en } \\
\text { pesos } \\
\text { al año }\end{array}$ & $\begin{array}{l}\% \text { del } \\
\text { tipo de } \\
\text { costo }\end{array}$ & \\
\hline \multirow[t]{6}{*}{ Directo } & Medicamentos & 58.862 & 69 & 50.211 & 70 & 111.727 & 70 & $<0,08$ \\
\hline & Consultas & 14.903 & 18 & 12.781 & 17 & 27.869 & 17 & $<0,01$ \\
\hline & Hospitalizaciones & 5.130 & 6 & 5.666 & 8 & 1.854 & 1 & NS \\
\hline & Radiografías & 3.544 & 4 & 1.783 & 2 & 14.303 & 9 & $<0,01$ \\
\hline & Kinesioterapia & 2.204 & 3 & 1.803 & 3 & 4.650 & 3 & NS \\
\hline & Total costo directo & \multicolumn{2}{|c|}{84.643} & \multicolumn{2}{|c|}{72.244} & \multicolumn{2}{|c|}{160.403} & $<0,04$ \\
\hline \multirow[t]{2}{*}{$\begin{array}{l}\text { Tipo de } \\
\text { costo }\end{array}$} & Variable & \multicolumn{2}{|c|}{$\begin{array}{l}\text { Población en } \\
\text { seguimiento }\end{array}$} & \multicolumn{2}{|c|}{$\begin{array}{c}\text { Población con asma } \\
\text { no severo }\end{array}$} & \multicolumn{2}{|c|}{$\begin{array}{c}\text { Población con asma } \\
\text { severo }\end{array}$} & $\mathbf{p}$ \\
\hline & & $\begin{array}{l}\text { Prome- } \\
\text { dio en } \\
\text { pesos } \\
\text { al año }\end{array}$ & $\begin{array}{l}\text { Promedio } \\
\text { días/año }\end{array}$ & $\begin{array}{c}\text { Prome- } \\
\text { dio en } \\
\text { pesos } \\
\text { al año }\end{array}$ & $\begin{array}{l}\text { Promedio } \\
\text { días/año }\end{array}$ & $\begin{array}{l}\text { Prome- } \\
\text { dio en } \\
\text { pesos } \\
\text { al año }\end{array}$ & $\begin{array}{l}\text { Promedio } \\
\text { días/año }\end{array}$ & \\
\hline \multirow[t]{3}{*}{ Indirecto } & Ausentismo laboral & 3.038 & $0,42 \pm 1,8$ & 3.010 & $0,41 \pm 1,9$ & 3.200 & $0,44 \pm 0,8$ & NS \\
\hline & Ausentismo escolar & 18.869 & $12,7 \pm 14,7$ & 15.303 & $10,3 \pm 10,1$ & 40.710 & $27,4 \pm 27$ & $<0,001$ \\
\hline & $\begin{array}{l}\text { Total costo } \\
\text { indirecto }\end{array}$ & 21.907 & & 18.313 & & 43.910 & & $<0,01$ \\
\hline $\begin{array}{l}\text { Total } \\
\text { costo }\end{array}$ & & 106.550 & & 90.557 & & 204.313 & & $<0,05$ \\
\hline
\end{tabular}

con asma severa en consultas y radiografías $(\mathrm{p}<$ $0,01)$. No se alcanzó significación estadística al comparar el ítem medicamentos $(\mathrm{p}<0,08)$. Los costos indirectos fueron $\$ 21.907$, calculados en base a la inasistencia laboral del cuidador y el costo por día de ausentismo escolar según subvención ministerial.

\section{Discusión}

Esta investigación se centró en la APS, en un sector de alta vulnerabilidad social. La "Guía clínica para el diagnóstico y tratamiento del asma en el menor de 15 años" del Ministerio de Salud de Chile $^{29}$ lo categoriza según frecuencia y severidad 
de las crisis en leve o episódico infrecuente, moderado o episódico frecuente y severo o persistente. Las guías GINA adicionan elementos de medición objetiva de la función pulmonar y la intensidad de la interferencia de los síntomas con la actividad del paciente. Sin embargo, por aspectos prácticos, en la APS se decidió ocupar una categorización clínica y no funcional de severidad del asma, diferenciando asma severo, cuando hubo reconocimiento en un videocuestionario ${ }^{18,19}$ de un "episodio severo" de asma agudo. Dado que este hecho es un apropiado y práctico elemento clínico en escolares ${ }^{5,17,30}$, siendo muy probable que un videocuestionario tenga ventajas sobre otros elementos clínicos para interpretar la realidad de la enfermedad ${ }^{19,31}$.

Los pacientes asmáticos generaron globalmente más del doble de consultas en APS que la población general del mismo grupo etáreo ${ }^{32}$. Más de $80 \%$ de las consultas debidas a síntomas respiratorios se efectuaron en la APS, lo que remarca el protagonismo de este nivel de atención en el manejo de la enfermedad y sus exacerbaciones. Sin embargo, las consultas realizadas en escenarios de urgencia suman un tercio del total; recalcando la importancia de las exacerbaciones como característica clínica en niños y adolescentes. Estos hechos también podrían explicar el elevado uso de broncodilatadores de rescate.

Menos de la mitad de los pacientes, usó CI regularmente, incluso en aquellos con asma severo. Este hecho, junto con el uso frecuente de broncodilatadores de rescate, podría relacionarse a mal control del asma vinculado a determinantes como educación, comprensión del paciente/cuidador insuficiente o ambos.

Dichos aspectos se recalcan en las guías internacionales de recomendación del manejo de asma recientemente publicadas ${ }^{13,29}$.

La metodología de costos utilizada en este estudio es conservadora. La evaluación no consideró costos variables atribuibles a farmacia e insumos utilizados en los escenarios de hospitalización y servicios de urgencia. Representa en términos generales un valor base, ya que para su obtención se utilizaron los precios de los fármacos más bajos ofertados por el mercado en compra directa. No se utilizó el referente de compras centralizadas por el Ministerio de Salud para medicamentos de la canasta de asma, dado que se incluyó además medicamentos para el control de rinitis alérgica (comorbilidad frecuente), no contemplados en dicha canasta.

Este estudio demostró que los costos directos están principalmente determinados por los medicamentos y consultas y, en menor medida, por los exámenes, procedimientos y hospitalizaciones. Esta distribución distinta a la obtenida en estudios de fármaco-economía de Estados Unidos de Norteamérica y Europa (donde mayores costos se vinculan a exacerbaciones que requieren consultas en los servicios de urgencia y hospitalizaciones) ${ }^{11,12}$, probablemente se relaciona con el bajo número de pacientes con asma severa que se controlan en APS. No obstante, es la representación real del escenario en que se realizó el estudio y las cifras obtenidas duplican el per cápita anual asignado para la comuna.

Al calcular los costos indirectos en función del ausentismo laboral de los cuidadores, al ser las madres, mayoritariamente sin trabajo remunerado, quienes ejercen dicho rol, el impacto de la enfermedad tiende a ser muy escaso en el costo total estimado. Aunque el ausentismo escolar no representa un gasto del sistema de salud, expresa la pérdida de un beneficio social y una merma en el desempeño académico del niño. Aunque el impacto familiar que el ausentismo escolar tiene en los costos indirectos es difícil de evaluar, los pacientes severos prácticamente triplican las cifras promedios del grupo en su conjunto, sobrepasando el límite de inasistencia permitido para aprobar por el MINEDUC (26 días/año) y por lo tanto, requieren un análisis intersectorial. Aunque no puede desprenderse directamente de esta investigación, el ausentismo podría vincularse a pobre control de la enfermedad.

El asma impacta significativamente la CVRS. De las distintas dimensiones evaluadas, el área emocional es la que tiene mejor percepción y la limitación de las actividades diarias la peor. La misma tendencia puede observarse en estudios europeos $^{16,33}$. La severidad del asma profundiza estos hallazgos en las tres dimensiones evaluadas, pero aquella vinculada a síntomas es la que cobra mayor significación estadística.

La información descriptiva de este estudio debe ser interpretada con cautela. La principal limitación fue la discreta participación de la población, reflejada en una elevada pérdida de los pacientes durante el seguimiento, impidiendo generalizar resultados al conjunto de pacientes en control en 
Impacto del asma en escolares de dos centros de salud primaria - C. Velástegui et al

APS. Aunque menos de la mitad de los pacientes completaron el estudio, no hubo diferencias en edad y genero entre ellos. Sin embargo, el desbalance de menor severidad en el grupo que finalizo el estudio podría significar un menor impacto del asma en las variables analizadas. El consentimiento informado explicitaba la libertad de abandonar el estudio y no hubo incentivo a los niños ni sus familias para asegurar su permanencia.

Parece importante, que futuros estudios, con diseños similares, pero con un mayor número de pacientes puedan corroborar estos hallazgos. Del mismo modo, hacen falta estudios que exploren la validez de estos resultados en otras realidades socioeconómicas. La elección de realizar una investigación de estas características, con las limitaciones y sesgos señalados, parece interesante en atención a que el área suroriente congrega aproximadamente un tercio de la población del sistema público de salud de la $\mathrm{RM}^{34}$ representando un importante grupo de beneficiarios con altos índices de vulnerabilidad social como lo demuestran los resultados socio-demográficos descritos, con baja escolaridad y pobre inserción laboral materna. Estos antecedentes adquieren relevancia, dado que la madre es quien frecuentemente ejerce el rol de cuidador de los pacientes pediátricos.

Los aspectos considerados en este estudio permiten señalar que el asma, en escolares atendidos en APS, afecta su percepción de bienestar en salud, asociándose a mayor morbilidad y mayores costos sanitarios. Dado que la mayoría de los pacientes corresponde a asma no-severo es posible especular que el control de la enfermedad no guarda estricta relación con la categorización de severidad ${ }^{35,36}$, y que pese a que el asma severo, tiene peor CVRS y mayores gastos, los pacientes menos severos también tienen indicadores de asma mal controlado, evidenciado en el alto uso de broncodilatadores de rescate. Estos resultados son congruentes con lo reportado en la literatura nacional ${ }^{37}$.

Conclusiones: Los escolares asmáticos evaluados en este estudio presentan un deterioro en su CVRS, mayor morbilidad y alto costo sanitario y personal, lo que se observa en mayor grado en pacientes con asma severa. La caracterización clínica del asma en este grupo sugiere que el uso de medicamentos controladores y su correcta y oportuna administración deben ser reforzados y probablemente incluidos en un plan de accióneducación. Sin embargo, las estrategias programá- ticas que deben implementarse para perfeccionar el manejo del asma en la APS requerirán abordar múltiples dominios y condicionantes en investigaciones futuras.

Agradecimientos: Los autores expresan su agradecimiento a la Dra. Solange Caussade, Pediatra Broncopulmonar de la Pontificia Universidad Católica de Chile, por la facilitación de material para este trabajo. Finalmente agradecemos a los Dres. Tomás Pantoja C. y Mildred Rojas, directores de los Centros de Salud Familiar donde se llevó a cabo esta investigación, como a todo su personal que colaboró desinteresadamente en la misma.

\section{Referencias}

1. Von Mutis E. The burden of childhood asthma. Arch Dis Child 2000; 82 (SupplI): ii2-5.

2. Fischer GB, Camargos PA, Mocelin HT. The burden of asthma in children: a Latin American perspective. Paed Resp Rev 2005; 6: 8-13.

3. Sennhauser F. Burden of asthma in children: a European perspective. Paed Resp Rev. 2005; 6: 2-7.

4. Sidney S. BRAMAN. The Global Burden of Asthma. CHEST 2006; 130: 4S-12S.

5. Mallol J, Aguirre V, Aguilar P, Calvo M, Amarales L, Arellano P, et al. Cambios en prevalencia de asma en escolares chilenos entre 1994 y 2002. Rev Med Chile 2007; 135: 580-6.

6. Lozano P, Fishman P, Vonkorff M, Hecht J. Health Care Utilization and Cost Among Children With Asthma. Pediatrics 1997; 99: 757-64.

7. Thomas M, Kocevar VS, Zhang Q, Yin DD, Price Dl. Asthma-Related Health Care Resource Use Among Asthmatic Children. Paediatrics 2005; 115: 129-34.

8. Forrest C, Starfield B, Riley A, Kang M. The Impact of Asthma on the Health Status of Adolescents. Pediatrics 1997; 99: 1-7.

9. Diette G, Markson L, Skinner E, Nguyen T, AlgattBergstrom P, Wu AW. Nocturnal Asthma in Children affects School Attendance, School Performance, and Parents 'work Attendance. Arch Pediatr Adolesc Med 2000; 154: 923-8.

10. Lloyd A, Pric D, Brown R. The impact of asthma exacerbations on health-related quality of life in moderate to severe asthma patients in the UK. Prim Care Resp J. 2007; 16: 22-7.

11. Kocevar VS, Bisgaard H, Jönsson L, Valovirta E, Kristensen F, Yin DD, et al. Variations in Paediatric Asthma Hospitalization Rates and Costs Between and Within 
Impacto del asma en escolares de dos centros de salud primaria - C. Velástegui et al

Nordic Countries. CHEST 2004; 125: 1680-4.

12. Ungar W, Coyte P. Prospective Study of the Patient-Level Cost of Asthma Care in Children. Pediatr Pulmonol 2001; 32: 101-8.

13. Global Initiative for Asthma, (GINA). Global Burden of Asthma. Disponible en www.ginasthma.com [Consultado el 10 de marzo de 2008].

14. Bacharier Lb, Boner A, Carlsen Kh, Eigenmann Pa, Frischer T, Gôtz M, et al. European Pediatric Asthma Group. Diagnosis and treatment of asthma in childhood: a PRACTALL consensus report. Allergy 2008; 63: 5-34.

15. Wolf FM, Guevara JP, Grumcm, Clark NM, Cates CJ. Educational interventions for asthma in children. Cochrane Database Syst Rev 2003; CD000326.

16. González P, Del Castillo L, García Jl, Ramírez C, Lahoz B, Barros C. Calidad de vida en una población de niños asmáticos. Aten Primaria 2006; 38: 96-101.

17. Mallol J, Cortez E, Amarales L, Sánchez I, Calvo M, Soto S, et al. [Prevalence of asthma in Chilean students. Descriptive study of 24470 children. ISAAC-Chile]. Rev Med Chile 2000; 128: 279-85.

18. Asher MI, Keil U, Anderson HR, Beasley R, Crane J, Martínez F, et al. International Study of Asthma and Allergies in Childhood (ISAAC): rationale and methods. Eur Respir J 1995; 8: 483-91.

19. Crane J, Mallol J, Beasley R, Stewart A, Asher Mi, on Behalf of the International Study of Asthma and Allergies in Childhood (ISAAC) Phase I Study Group. Agreement between written and video questions for comparing asthma symptoms in ISAAC. Eur Rerspir J 2003; 21: 455-61.

20. Juniper EF, Guyatt GH, Fenny DH, Ferrie PJ, Griffith LE, Townsend M. Measuring quality of life in children with asthma. Quality of Life Research 1996; 5: 35-46.

21. La Scala C, Naspitz C, Solé D. Adaptation and validation of the Pediatric Asthma Quality of Life Questionnaire (PAQLQ) in Brazilian asthmatic children and adolescents. J Pediatr (Rio J) 2005; 81: 54-60.

22. Elizabeth C, Suzanna S, Tim C F, Chi S L, Mital R, BeeWah L. Pediatric asthma quality of life questionnaire: validation in children from Singapore. Asian Pac J Allergy Immunol 1999; 17: 155-61.

23. Badia X, García-Hernández G, Cobos N, López-David C, Nocea G, Roset M. Validity of the Spanish version of the Pediatric Quality of Life Questionnaire for evaluating quality of life in asthmatic children. Med Clin (Barc) 2001; 116: 565-72.

24. Vidal A, Duffau G, Ubilla C. Calidad de vida en el niño asmático y su cuidador. Rev Chil Enf Respir 2007; 23: 160-6.

25. FONASA. Arancel de Prestaciones de Salud 2008.
Disponible en: http://www.fonasa.cl/prontus_fonasa/ antialone.html?page $=$ http://www.fonasa.cl/prontus_fonasa/site/artic/20060310/pags/20060310112139.html [Consultado el 10 de marzo de 2008].

26. DIPRES-Gobierno de Chile. Programa de Reforzamiento de la Atención Primaria de Salud (PRAPS). Disponible en: http://www.dipres.cl/574/propertyvalue-15884.html [Consultado el 10 de marzo de 2008].

27. Senado-República de Chile, Ley 20.204. Disponible en: http://www.senado.cl/appsenado/index.php?mo= proyectos\&ac=leyes [Consultado el 22 de enero de 2009].

28. Comunidad Escolar-MINEDUC. Santiago: Coordinación Nacional de Subvenciones del Ministerio de Educación. Disponible en: www.comunidadescolar.cl/ documentacion/Subvencion/Enero2008webValoresSubvenciones.pdf. [Consultado el 22 de enero del 2009].

29. Ministerio de Salud. Diagnóstico y tratamiento del asma en el menor de 15 años, 2006. Santiago de Chile: Gobierno de Chile, 2006.

30. Valdivia G, Caussade S, Navarro H, Cerda J, Pérez E, et al. Influencia del nivel socioeconómico en el asma bronquial y cambios en su prevalencia en población escolar en un período de 6 años. Rev Méd Chile 2009; 137: 215-25.

31. Pizzichini MM, Rennie D, Senthilselvan A, Taylor B, Habbick BF, Sears MR. Limited agreement between written and video asthma symptom questionnaires. Pediatr Pulmonol 2000; 30: 307-12.

32. Médicos Atención Primaria. IV Congreso Nacional de Médicos de Atención Primaria: Conferencia "Atención Primaria de Salud. Perspectivas de Desarrollo. Ministra de Salud, Soledad Barria". Disponible en http:// www.medicosaps.cl/IV\%20Congreso\%20Nacional\%20 M\%E9dicos\%20APS/Conferencias/MEDICOS\%20APs. pdf. [Consultado el 11 de febrero del 2009].

33. Reichenberg K. Quality of life in childhood asthma. Acta Paediatr 2000; 89: 989-95.

34. Fondo Nacional de Salud (FONASA). Estadísticas: Demografía. Disponible en https://fon.fonasa.cl/prontus_fonasa/site/artic/20070112/asocfile/01_demografia_pagina_web_15_09_2008_ja.xls. [Consultado el 10 de febrero del 2009].

35. Yawn B, Brenneman S, Allen-Ramey F, Cabana M, Markson L. Assessment of Asthma Severity and Asthma Control in Children. Pediatrics 2006; 118: 322-9.

36. Humbert M, Holgate S, Boulet L, Bousquet J. Asthma control or severity: that is the question. Allergy 2007; 62: 95-101.

37. Vidal A, Ubilla C, Duffau G. Control de asma en adolescentes. Rev Med Chile 2008; 136: 859-66. 